\title{
439.
}

\section{ADDITION À LA NOTE SUR QUELQUES TORSES SEXTIQUES.}

[From the Annali di Matematica pura ed applicata, tom. II. (1868), pp. 219-221.]

$J_{E}$ viens de trouver ce que signifie la condition $J=0$. Considérons deux surfaces quadriques qui se touchent (d'un contact ordinaire). Les équations tangentielles peuvent s'écrire sous la forme

$$
\begin{aligned}
& a \xi^{2}+b \eta^{2}+c \zeta^{2}+2 n \zeta \omega=0, \\
& a^{\prime} \xi^{2}+b^{\prime} \eta^{2}+c^{\prime} \xi^{2}+2 n^{\prime} \zeta \omega=0,
\end{aligned}
$$

et l'on satisfait à ces équations par des valeurs de $-\xi, \eta, \zeta, \omega$ qui contiennent un paramètre arbitraire $\theta$, en écrivant

$$
\xi: \eta: \zeta: \omega=\alpha\left(\theta+\frac{1}{\theta}\right): \beta\left(\theta-\frac{1}{\theta}\right): \epsilon: \gamma\left(\theta^{2}+\frac{1}{\theta^{2}}\right)+\delta
$$

en effet cela donne

$$
\begin{array}{ll}
a \alpha^{2}+b \beta^{2}+2 n \gamma \epsilon=0, & 2 a \alpha^{2}-2 b \beta^{2}+c \epsilon^{2}+2 n \delta \epsilon=0, \\
a^{\prime} \alpha^{2}+b^{\prime} \beta^{2}+2 n^{\prime} \gamma \epsilon=0, & 2 a^{\prime} \alpha^{2}-2 b^{\prime} \beta^{2}+c^{\prime} \epsilon^{2}+2 n^{\prime} \delta \epsilon=0,
\end{array}
$$

ce qui détermine les valeurs de $\alpha: \beta: \gamma: \delta: \epsilon$. L'équation du plan tangent commun sera donc

$$
x \alpha\left(\theta+\frac{1}{\theta}\right)+y \beta\left(\theta-\frac{1}{\theta}\right)+z \epsilon+w\left[\gamma\left(\theta^{2}+\frac{1}{\theta^{2}}\right)+\delta\right]=0,
$$

ou, en multipliant par $12 \theta^{2}$, cette équation sera

$$
(a, b, c, d, e \gamma \theta, 1)^{4}=0,
$$


les valeurs des coefficients étant

$$
\begin{aligned}
& a=12 \gamma w \\
& b=3(\alpha x+\beta y) \\
& c=2(\delta w+\epsilon z) \\
& d=3(\alpha x-\beta y), \\
& e=12 \gamma w .
\end{aligned}
$$

En représentant par $A a+4 B b+6 C c+4 D d+E e=0$ l'équation qui lie les fonctions linéaires $a, b, c, d$, e cette équation sera $a-e=0$; on a donc $A=-E=1, B=C=D=0$ : l'invariant $J$ de la fonction $(A, B, C, D, E \gamma \tau, 1)^{4}$ est donc $=0$.

Nous arrivons ainsi à la conclusion que la torse sextique

$$
\left(a e-4 b d+3 c^{2}\right)^{3}-27\left(a c e-a d^{2}-b^{2} e-c^{3}+2 b c d\right)^{2}=0,
$$

où les fonctions linéaires $a, b, c, d, e$ sont liées par une équation

$$
A a+4 B b+6 C c+4 D d+E e=0,
$$

telle que l'invariant

$$
J=A C E-A D^{2}-E B^{2}-C^{3}-2 B C D
$$

de la fonction $(A, B, C, D, E \gamma \tau, 1)^{4}$ est $=0$ (cas $7^{\circ}$ de la Note), est la torsc enveloppée par le plan tangent commun de deux surfaces quadriques qui se touchent d'un contact ordinaire. J'ai trouvé l'équation de cette torse dans le Mémoire, "On the Developable Surfaces which arise from two Surfaces of the Second Order," Camb. and Dubl. Math. Jour., t. v. (1850), pp. 46-57, voir p. 56, [85]: $a, b, c, n,-a^{\prime}, b^{\prime}, c^{\prime}, n^{\prime}$ y dénotent les mêmes coefficients comme à présent, et en écrivant

$$
\begin{aligned}
& b c^{\prime}-b^{\prime} c=f, \quad a n^{\prime}-a^{\prime} n=p, \\
& c a^{\prime}-c^{\prime} a=g, \quad b n^{\prime}-b^{\prime} n=q, \\
& u b^{\prime}-a^{\prime} b=h, \quad c n^{\prime}-c^{\prime} n=r,
\end{aligned}
$$

(et de là $p f+q g+r h=0)$, l'équation trouvée est

$$
f^{2} g^{2} h^{2} w^{6} \ldots+4 p q r\left(q x^{2}+p y^{2}\right)^{3}=0 .
$$

Je vais vérifier ces termes. Partant de l'équation $(a, b, c, d, e \gamma \theta, 1)^{4}=1$, l'équation de la torse, en y introduisant pour commodité le facteur $-\frac{1}{216} p q r$, sera

$$
\begin{gathered}
-\frac{1}{216} p q r\left\{\left[4\left[(\delta w+\epsilon z)^{2}+12 \gamma^{2} w^{2}-3\left(\alpha^{2} x^{2}-\beta^{2} y^{2}\right)\right]^{3}\right.\right. \\
\left.-\left[2(\delta w+\epsilon z)^{3}-9(\delta w+\epsilon z)\left(\alpha^{2} x^{2}-\beta^{2} y^{2}\right)-27(\delta w+\epsilon z) \gamma^{2} w^{2}+54\left(\alpha^{2} x^{2}+\beta^{2} y^{2}\right) \gamma w\right]^{2}\right\}=0 .
\end{gathered}
$$


En prenant $\gamma \epsilon=a b^{\prime}-a^{\prime} b=h$, on obtient pour $\alpha, \beta, \gamma, \delta, \epsilon$ les valeurs

et de là

$$
\alpha^{2}=2 q, \quad \beta^{2}=-2 p, \quad \gamma \epsilon=h, \quad \epsilon^{2}--\frac{8 p q}{r}, \quad \delta \epsilon=\frac{2(f p-g q)}{r},
$$

$$
\gamma^{2}=-\frac{h^{2} r}{8 p q}, \quad \delta^{2}=-\frac{(f p-g q)^{2}}{2 p q r}=-\frac{h^{2} r^{2}+4 f g p q}{2 p q r}=4 \gamma^{2}+\frac{2 f g}{r}, \quad \delta^{2}-4 \gamma^{2}=\frac{2 f g}{r}
$$

Les termes en $w^{6}$ et $\left(x^{2}, y^{2}\right)^{3}$ sont

$$
\begin{aligned}
& -\frac{1}{216} p q r\left\{\left[4\left(\delta^{2}+12 \gamma^{2}\right)^{3}-\left(2 \delta^{*}-72 \gamma^{2} \delta\right)^{2}\right] w^{6}-108\left(\alpha^{2} x^{2}-\beta^{2} y^{2}\right)^{3}\right\}, \\
= & -\frac{1}{216} p q r\left\{432 \gamma^{2}\left(\delta^{2}-4 \gamma^{2}\right)^{2} w^{6}-108\left(\alpha^{2} x^{2}-\beta^{2} y^{2}\right)^{3}\right\}
\end{aligned}
$$

ces termes sont donc

$$
\begin{aligned}
& =-\frac{1}{216} p q r\left\{-432 \frac{h^{2} r}{8 p q} \cdot \frac{4 f^{2} g^{2}}{r^{2}} w^{2}+864\left(q x^{2}+p q^{2}\right)^{2}\right\} \\
& =f^{2} g^{2} h^{2} w^{6}+4 p q r\left(q x^{2}+p y^{2}\right)^{3}
\end{aligned}
$$

comme cela doit être.

Cambridge, le 22 septembre 1868. 